\title{
Nota científica \\ PIONERO-INIA: EL PRIMER TRIGO DOBLE PROPÓSITO PARA CHILE
}

\section{PIONERO-INIA: THE FIRST DOUBLE PURPOSE WHEAT FOR CHILE}

\author{
Claudio Jobet ${ }^{1,2 *}$, Volker Lein ${ }^{3}$, Ricardo Madariaga ${ }^{4}$, Iván Matus ${ }^{4}$, Dalma Castillo ${ }^{4}$, y Javier Zuñiga ${ }^{1}$ \\ ${ }^{1}$ Instituto de Investigaciones Agropecuarias (INIA), Centro Regional Carillanca, Casilla 58-D, Temuco, \\ Chile. \\ ${ }^{2}$ Universidad de La Frontera, Facultad de Agronomía, Casilla 54-D, Temuco, Chile. \\ ${ }^{3}$ Saaten Union Research, Francia. \\ ${ }^{4}$ Instituto de Investigaciones Agropecuarias (INIA), Centro Regional Quilamapu, Casilla 58-D, \\ Chillán, Chile. \\ * Autor para correspondencia E-mail: cjobet@inia.cl
}

\section{RESUMEN}

Pionero INIA es la primera variedad de trigo doble propósito liberado por el Programa Nacional de Trigo del INIA Carillanca. Es una variedad de invierno (Triticum aestivum L.) producto de la selección de germoplasma recibido de la Empresa Saaten Union Research de Francia. Mostró excelente adaptabilidad para siembras tempranas, en zonas de inviernos largos y primaveras húmedas. La altura promedio de planta adulta alcanza $\operatorname{los} 90 \mathrm{~cm}$, siendo clasificado como una variedad de trigo enano a semi enano. El rendimiento promedio, en diferentes localidades y varios años de evaluación, fue significativamente superior al testigo Kumpa INIA, destacándose por su resistencia a enfermedades y tipo agronómico. Sus características de calidad molinera y de almidón permiten utilizarlo en la industria galletera, ganadera y acuícola.

Palabras clave: cultivar, trigo invierno, rendimiento, sintético, alimentación animal.

\section{ABSTRACT}

Pionero INIA is the first double purpose wheat cultivar released in Chile by the National Wheat Program from INIA Carillanca. It is a winter wheat variety (Triticum aestivum L.) obtained from a germplasm selected from the Saaten Union Research Company of France. It showed excellent adaptability to agro-climatic zones with early sowing, long winters and wet spring. The adult plant reached a mean height of $90 \mathrm{~cm}$, so that Pionero INIA can be classified as a semi-dwarf to dwarf wheat variety. The mean grain yield was significantly higher than the control, Kumpa INIA, for the different locations and years of evaluation, while its performance was particularly higher in terms of disease resistance and agronomic type. Its milling and starch quality characteristics allow for its use in the cookie industry, as well as in the animal and fish feed manufacturing industries.

Key words: cultivar, winter wheat, yield, synthetic, animal feed. 


\section{INTRODUCCION}

Pionero INIA es una variedad de trigo (Triticum aestivum L.) de hábito de desarrollo invernal, con bajos requerimientos de frío, perteneciente al Instituto de Investigaciones Agropecuarias, INIA, seleccionado a partir de germoplasma avanzado recibido de la Empresa Saaten Union Research, de Francia. Este trigo fue recibido en un jardín $\mathrm{F}_{10}$ el año 2006, con la denominación interna EssaiSUR.400/12. Su origen fue producto de una cruza compuesta realizada en el programa de trigos híbridos de esta empresa, cuyos progenitores fueron cinco genotipos elite mas dos líneas sintéticas mejoradas derivadas del Triticum dicoccum (AABB), especie ancestral del Triticum durum (AABB) y de Triticum aestivum (AABBDD), pertenecientes a la Universidad de Wageningen, Holanda, las cuales se utilizaron como fuente donantes de genes para tolerancia a stress biótico y abiótico, originadas a partir de cruzas amplias con T. dicoccum (Emmer) con el fin de incluir, preferentemente, genes de resistencia a enfermedades en trigo (Volker Lain, Director Científico, Saaten Union Research, Francia. Comunicación personal). La génesis de Pionero INIA se asemeja a la descripción establecida por Jobet et al. (2017) para la variedad de trigo de invierno denominada Maxwell.

Pionero INIA fue evaluado en Chile durante varias temporadas, desde la Región de la

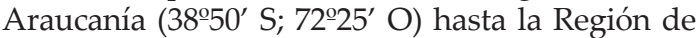
Los Lagos (40 51' S; 7312' O), destacándose por su tipo agronómico, baja altura de planta, buena resistencia a la tendedura, excelente sanidad y gran potencial productivo. Desde el punto de vista nutricional tiene un alto tenor en almidón, tanto en cantidad como en calidad, característica importante para ser utilizada en la alimentación de bovinos y salmones. Si bien es cierto, no existen estadísticas oficiales respecto al uso del trigo como componente en la dieta de animales, la información publicada indica que un $9 \%$ de la producción de la Región de Los Ríos y de Los Lagos en el sur del país se utiliza para tal efecto (Mellado, 2007). Por otro lado, las características de calidad industrial permiten establecer que esta variedad se comporta como un trigo de tipo galletero (clase suave), con un bajo valor de gluten húmedo, un grano de textura blanda y un valor de $\mathrm{W}$ (alveograma) no superior a 100, presentando una curva de gran extensibilidad y cuya relación $\mathrm{P} / \mathrm{L}$ es menor a 0,7 . Ambas características, nutricionales e industriales, hacen que este trigo sea considerado como el primer trigo doble propósito liberado en Chile, apto para ser utilizado en las industrias molinera y ganadera.

\section{ANTECEDENTES DE PIONERO INIA}

Esta variedad ha sido evaluada desde el 2007 en ensayos de rendimiento preliminar en Vilcún,

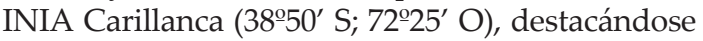
por su tipo agronómico, adaptabilidad a zonas de siembras tempranas e inviernos largos, alto nivel productivo y buen comportamiento fitosanitario. Posteriormente a partir del año 2009 se incorporó a los ensayos estándares nacionales, involucrando

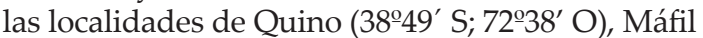
$\left(35^{\circ} 39^{\prime} \mathrm{S} ; 73^{\circ} 02^{\prime} \mathrm{O}\right)$ y Purranque ( $40^{\circ} 51^{\prime} \mathrm{S}$; $73^{\circ} 12^{\prime} \mathrm{O}$ ), lo que representa a tres regiones del sur de Chile, donde se concentra mas del $50 \%$ de la superficie sembrada de trigo, y un porcentaje mayor aun de la actividad ganadera y acuícola del país (ODEPA, 2017).

\section{DESCRIPCIÓN MORFOLOGICA DE LA PLANTA}

La planta tiene tallo hueco y baja altura, entre 75 a $90 \mathrm{~cm}$, con buena resistencia a la tendedura. Su período vegetativo desde la siembra a espigadura es alrededor de ocho a diez días más precoz que la variedad de invierno Kumpa INIA, y similar a la variedad Maxwell en INIA Carillanca, Vilcún (Región de La Araucanía). Cuando se siembra el 20 de mayo en INIA Carillanca, Vilcún, espiga entre 24 y 26 de noviembre (187 días aproximadamente de siembra a espigadura). En Máfil (Región de Los Ríos) y Purranque (Región de Los Lagos), sembrado el 23 de mayo, espiga alrededor del 30 de noviembre (172 y 170 días aproximadamente de siembra a espigadura, respectivamente), siendo alrededor de 11 días más precoz que Kumpa INIA y dos días más tardío que Maxwell. Durante ese período de tiempo reúne entre 720 a 780 grados días base $5^{\circ} \mathrm{C}$, en Vilcún, y entre $757 \mathrm{y}$ 854 grados días base $5^{\circ} \mathrm{C}$ en Purranque. Su hábito de crecimiento a la macolla es muy rastrero y sus aurículas no presentan antocianinas. La lámina de la hoja presenta glaucosidad muy débil o ausente al igual que el tallo. La espiga es de color blanco, forma paralela, densidad alta, con barbas ausentes y semierecta a la madurez. Las glumas muestran un hombro derecho, de ancho medio, diente obtuso y vellosidad externa ausente. El grano es ovoide a semi-alargado, de tamaño mediano, color rojo, con cepillo ausente o muy corto, surco central marcado y un peso promedio de $40 \mathrm{~g}$ los 1000 granos, con un rango de 33,0 a 42,0 g, dependiendo de la época de siembra y localidad.

\section{CARACTERISTICAS FITOPATOLOGICAS}

Pionero INIA se destaca por su resistencia al polvillo estriado causado por Puccinia striiformis 
West. f. sp. tritici Erikss., sin embargo, a través de los años, ha ido presentado cierta sensibilidad a polvillo colorado de la hoja producido por Puccinia triticina Erikss. Tiene buena tolerancia a septoriosis (Mycosphaerella graminicola (Fuckel) J. Schröt.), es resistente a oidio causado por el hongo Blumeria graminis D.C. f sp. tritici Marchal. Un aspecto importante de considerar es su sensibilidad a enfermedades radiculares como mal de pie (Gaeumannomycea graminis var. tritici), por lo que se recomienda sembrarla en rotaciones adecuadas y en suelos libres de esta enfermedad. Sus reacciones de resistencia y sensibilidad (Saari, y Prescott, 1975; Peterson et al., 1984), a estas enfermedades podrían estar relacionadas a su génesis genética, principalmente por aquellos genes provenientes de uno de sus progenitores, Triticum dicoccum (AABB).

\section{RENDIMIENTO DE GRANO}

Pionero INIA fue incorporado inicialmente en un ensayo agronómico preliminar junto a otras veinte variedades comerciales y líneas avanzadas en Vilcún (INIA Carillanca); y posteriormente, en la temporada 2009/2010, se incorporó a los ensayos estándar realizados en cuatro localidades, Quino, Vilcún, Máfil y Purranque, que abarcan a tres regiones del sur del país. Los ensayos estuvieron estructurados con un diseño de bloques completos (genotipos) al azar con cuatro repeticiones, en parcelas de $6 \mathrm{~m}^{2}$, cuyo manejo agronómico fue óptimo, con el fin de detectar diferencias entre genotipos; la fertilización base fue entre 180 a 220 $\mathrm{kg} \mathrm{ha}^{-1}$ de N, 120 a $140 \mathrm{~kg} \mathrm{ha}^{-1}$ de $\mathrm{P}_{2} \mathrm{O}_{5}$ y entre 60 a $80 \mathrm{~kg} \mathrm{ha}^{-1}$ de $\mathrm{K}_{2} \mathrm{O}$, según la localidad y análisis de suelo. Una vez cosechada la parcela completa para cada localidad se realizó un Análisis de la Varianza (ANDEVA) y para verificar las diferencias entre los promedios de los tratamientos se utilizó la Prueba de Rangos Múltiples de Tuckey $(\mathrm{p}<0,05)$.

En las cuatro localidades Pionero INIA presentó un buen potencial productivo, promedio de 12,9 ton ha- ${ }^{-1}$, superior a Kumpa INIA en 2,24 ton ha ${ }^{-1}$ (Tabla 1), alcanzando diferencias estadísticamente significativas entre ambas para algunas localidades y temporadas, con altos rendimientos e incluso superiores a testigos comerciales (datos no presentados). Kumpa INIA se consideró el testigo más adecuado ya que era la variedad de invierno mas demandada por los agricultores del sur de Chile.

Como una forma de conocer su nivel productivo en las distintas regiones del sur del país, Pionero INIA fue evaluado desde el año 2009 hasta el año 2015, alcanzando un rendimiento promedio de 12,91 tha ${ }^{-1}$, con un máximo de 17,23 $\mathrm{t} \mathrm{ha}^{-1}$, siendo este uno de los más altos niveles productivos alcanzados en las regiones del sur. Es importante señalar que a nivel comercial, ha superado las 13,3 t ha ${ }^{-1}$ en la localidad de Máfil, transformándose en una alternativa muy interesante para los agricultores de esa zona (Semillas SEK, Comunicación personal, 2016). Los pesos del hectolitro son más bajos que los del trigo harinero, con valor promedio de 74,6 $\mathrm{kg} \mathrm{hL}^{-1}$ en comparación con Kumpa INIA que alcanzó los $79,2 \mathrm{~kg} \mathrm{hL}^{-1}$ (Tabla 2).

Tabla 1. Rendimiento promedio de grano $\left(\mathrm{t} \mathrm{ha}^{-1}\right)$ de Pionero INIA comparada con Kumpa INIA en cuatro localidades durante varias temporadas (2011/12 al 2014/15).

Table 1. Mean grain yield ( $\left(\mathrm{ha}^{-1}\right)$ of Pionero INIA variety compared to Kumpa INIA in four experimental sites over several seasons (2011/12 to 2014/2015).

\begin{tabular}{|c|c|c|c|c|c|c|}
\hline Localidad & Variedad & $\begin{array}{c}\text { Rendimiento } \\
2011 / 12\end{array}$ & $\begin{array}{c}\text { Rendimiento } \\
2012 / 13\end{array}$ & $\begin{array}{c}\text { Rendimiento } \\
2013 / 14\end{array}$ & $\begin{array}{c}\text { Rendimiento } \\
2014 / 15\end{array}$ & $\begin{array}{l}\text { Rendimiento } \\
\text { promedio }\end{array}$ \\
\hline & & & & --- tha $^{-1}$ & & -------- \\
\hline \multirow[t]{2}{*}{ Quino } & Pionero INIA & 10,21 & 17,27 & 13,52 & 14,52 & 13,88 \\
\hline & Kumpa INIA & 9,71 & 13,71 & 10,02 & 11,48 & 11,23 \\
\hline \multirow[t]{2}{*}{ Vilcún } & Pionero INIA & 9,54 & 10,41 & 14,52 & 11,10 & 11,39 \\
\hline & Kumpa INIA & 9,54 & 8,02 & 9,21 & 10,38 & 9,29 \\
\hline \multirow[t]{2}{*}{ Máfil } & Pionero INIA & 12,54 & 13,78 & 12,41 & 13,10 & 12,96 \\
\hline & Kumpa INIA & 11,58 & 11,35 & 10,80 & 10,85 & 11,14 \\
\hline \multirow[t]{2}{*}{ Purranque } & Pionero INIA & 12,01 & 9,89 & 17,23 & 14,56 & 13,42 \\
\hline & Kumpa INIA & 11,86 & 8,92 & 12,42 & 10,89 & 11,02 \\
\hline
\end{tabular}


Tabla 2. Características de calidad de Pionero INIA comparada con el testigo Kumpa INIA. Rangos promedios observados para diferentes localidades en dos temporadas.

Table 2. Quality characteristics of cv. Pionero INIA compared to control cv. Kumpa INIA. Mean values measured at different locations and in two seasons.

\begin{tabular}{|c|c|c|c|c|c|c|}
\hline \multirow[t]{3}{*}{ Características } & \multicolumn{6}{|c|}{ 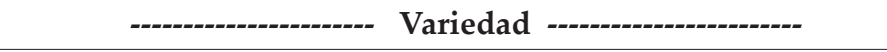 } \\
\hline & \multicolumn{3}{|c|}{------ Pionero INIA --- } & \multicolumn{3}{|c|}{-----Kumpa INIA ------ } \\
\hline & $2012 / 13^{*}$ & $2013 / 14^{*}$ & Prom. & $2012 / 13^{*}$ & $2013 / 14^{*}$ & Prom. \\
\hline Índice de dureza, $\%{ }^{(1)}$ & 24 & 25 & 24,5 & 20 & 18,7 & 19,4 \\
\hline Peso del hectolitro, $\mathrm{kg} \mathrm{hL}^{-1}$ & 76 & 76 & 76,0 & 81 & 81 & 81,0 \\
\hline Sedimentación Zeleny, cm(2) & 21 & 20 & 20,5 & 38 & 42,4 & 40,2 \\
\hline Proteína (N x 5,7), \% base seca & 8,1 & 9,2 & 8,7 & 11,4 & 11,0 & 11,2 \\
\hline Valor $\mathrm{W}^{(3)}$ & 78 & 62 & 70,0 & 168 & 212 & 190 \\
\hline $\mathrm{P}(\mathrm{m} / \mathrm{m})$ & 47 & 33 & 40,0 & 84 & 76 & 80 \\
\hline $\mathrm{L}(\mathrm{m} / \mathrm{m})$ & 76 & 86 & 81,0 & 83 & 83 & 83 \\
\hline $\mathrm{P} / \mathrm{L}$ & 0,6 & 0,4 & 0,5 & 1,0 & 1,0 & 1,0 \\
\hline Gluten húmedo, \% ${ }^{(4)}$ & 21,3 & 22,5 & 21,9 & 29,0 & 28,6 & 28,8 \\
\hline Falling Number, seg ${ }^{(5)}$ & 356 & 362 & 329 & 436 & 409 & 423 \\
\hline
\end{tabular}

* Laboratorio de Calidad de Trigo, INIA Carillanca.

(1) Índice de dureza: Valores de 16 a 25 corresponden a un grano duro; valores de 26 a 30 corresponden a un grano semiduro; valores de 31 o más corresponden a un grano blando.

(2) Sedimentación Zeleny: Cifras de 17 a $26,9 \mathrm{~cm}^{3}$ corresponden a un trigo suave; cifras de 27 a $32,9 \mathrm{~cm}^{3}$ corresponden a un trigo intermedio; cifras de 33 a $44 \mathrm{~cm}^{3}$ corresponde a un trigo fuerte; cifras superiores a $44 \mathrm{~cm}^{3}$ corresponde a un trigo muy fuerte.

(3) Valor W (alveograma): Menor de 150 corresponde a un valor bajo; entre 150 a 200 corresponde a un valor medio; más de 200 corresponde a un valor bueno

(4) Gluten húmedo: Igual o mayor a 30\% corresponde a trigo fuerte; entre 29,9 y 25,0\% corresponde a un trigo intermedio; contenido mínimo de $18,0 \%$ corresponde a un trigo suave.

(5) Falling Number: Índice de caída: Sobre 300 baja presencia de enzima alfa amilasa; bajo 170 alta presencia de enzima alfa amilasa.

\section{CALIDAD MOLINERA}

De acuerdo a los resultados de los análisis efectuados en el Laboratorio de Calidad de Trigos del INIA Carillanca (Tabla 2), Pionero INIA es un trigo de bajo peso del hectolitro $(76,0$ $\mathrm{kg} \mathrm{hL}^{-1}$ ) y de textura de grano blanda $(24,5)$. El porcentaje de proteína de grano es de $8,7 \%$, el valor de sedimentación $20,5 \mathrm{~mL}$, y el contenido de gluten húmedo cercano al 22,0\%, ubicándolo en la categoría de trigo suave (Instituto Nacional de Normalización, 2000). Sus características alveográficas son débiles, reflejado por su valor W (70,0 de promedio), indicando una mayor resistencia mecánica y menor tenacidad $(\mathrm{P} / \mathrm{L}=$ $0,5)$. Su harina de color blanco $\left(b^{*}=7,88\right)$, lo hace muy atractivo para la industria molinera, pero sin duda, en términos generales, su uso industrial está dirigido para la industria de la galleta y alimentación animal (Zúñiga J., Comunicación personal, 2016).

\section{POTENCIALIDAD DE PIONERO INIA COMO TRIGO DOBLE PROPOSITO}

El mejoramiento genético del trigo en Chile se ha hecho pensando en las características de una planta destinada a producir grano para consumo humano; sin embargo, alrededor del 9\%, o sea 141.000 toneladas (INE, 2014), de la cosecha 2013 se utilizó para suplementar raciones alimenticias para animales, especialmente vacas lecheras en la zona sur del país (Mellado, 2007). La superficie de siembra destinada para otros fines distintos al consumo humano es actualmente poco relevante, posiblemente porque no existe a la fecha un cultivar destinado a este uso específico. No obstante, es interesante referirse al uso del trigo como alimento para animales, dado que en algunas temporadas su consumo adquiere más importancia, y porque últimamente también se le usa en la elaboración de pellets para la alimentación de salmones. En los últimos años, y específicamente en las regiones del sur de 
Chile (Los Ríos y Los Lagos) el uso de trigo en las raciones de estos animales se ha transformado en actividades rutinarias, para lo cual ya existen informaciones respecto a demandas que alcanzan a mas de 8.000 toneladas de trigo (Integra, Osorno, Comunicación Personal, 2016). Paralelamente a ello, en Valdivia, algunas empresas relacionadas al rubro ganadero, han mostrado gran interés por contar con un trigo para alimentación animal adaptado al ambiente del sur y que reúna los requisitos de cantidad y calidad de almidón (Semillas SEK, Valdivia, Comunicación Personal, 2016).

\section{CONTENIDO DE ALMIDON}

De acuerdo a los resultados obtenidos en el Laboratorio de Biotecnología del INIA Carillanca, la variedad Pionero INIA alcanzó niveles de casi el $70 \%$ de almidón peso seco (Laboratorio de Bromatología, INIA Remehue, datos no mostrados), resultando similar al triticale $\mathrm{y}$ superando a todas las variedades comerciales de trigo-pan y a las especies alternativas de suplementación animal como avena y cebada. Esta característica es especialmente importante no solo para los ganaderos sino también gravita de forma significativa en la alimentación para salmones, lo que abre una demanda importante desde diversos frentes, para la región del sur de Chile.

La importancia del almidón es que es el mayor componente de la harina de trigo. En el endosperma de trigo existen tres tipos de almidones: de tamaño grande y lenticulares, de tamaño intermedio con forma ovoide y pequeños esféricos. De acuerdo con Hevia et al. (1986), los trigos con endosperma harinoso (blando), tienen gránulos muy grandes rodeados por una gran cantidad de gránulos pequeños, con poca matriz proteica, presentando una textura suelta, con espacios de aire entre ellos. Pionero INIA presenta un almidón formado preferentemente por gránulos grandes e intermedios, lo que se expresa en textura blanda, menor contenido en proteínas y por ende menor contenido de gluten, lo que le proporciona características más adecuadas para el consumo animal y para la elaboración de productos galleteros.

\section{PERFIL ELECTROFORÉTICO DE GLUTENINAS DE ALTO PESO MOLECULAR Y PRESENCIA DE TRANSLOCACIÓN 1BL.1RS.}

Los análisis de gluteninas efectuados mediante electroforesis de proteínas reducidas y alquiladas (Singh et al; 1991) muestran que Pionero INIA posee la combinación de alelos Glu1: nulo en el locus Glu-1A, 6 en el locus Glu-1B y 2+12 en el locus Glu-1D. La denominación de los alelos se efectuó según lo descrito por Payne y Lawrence (1983). El análisis de gliadinas efectuado según Bushuk y Zillman (1978) indica que Pionero INIA porta la translocación trigo-centeno 1BL.1RS. (Zuñiga et al., 2000).

\section{ANÁLISIS MOLECULARES}

El alelo de dureza Pinb-D1b, que se identifica por medio de la reacción en cadena de polimerasa (PCR), de acuerdo con la metodología de Zúñiga et al. (2001), no esta presente en Pionero INIA. Esto confirma el índice de dureza observado mediante espectroscopía NIR $(24,5)$ y avala el uso industrial de la variedad en alimentación animal y en la industria de la galleta. También los análisis moleculares respecto a la tolerancia a la acidez señalan que este cultivar porta el alelo TaALMT1, relacionado a la acidez, lo que podría indicar un cierto grado de sensibilidad a suelos ácidos.

\section{ZONA DE CULTIVO Y FECHAS DE SIEMBRA}

Por su ciclo semi precoz de desarrollo siembracosecha se recomienda sembrarlo temprano, en abril o temprano en mayo, en zonas de inviernos cortos y primaveras secas (suelos rojos arcillosos de la región de La Araucanía y zona de trumao en Osorno). Respecto a zonas del valle central y precordillera, con inviernos largos y primaveras húmedas, se recomienda sembrar Pionero INIA entre mediados de mayo y fines de junio.

\section{CONSIDERACIONES ESPECIALES}

1.- Es una variedad que ha mostrado moderada sensibilidad a suelos con problemas de acidez, por lo que se recomienda corregir esta situación mediante encalado.

2.- Tiene susceptibilidad a enfermedades radiculares por lo que se sugiere realizar una rotación de cultivos adecuada.

3.- Su sensibilidad a roya de la hoja hace necesario aplicar un fungicida para evitar un posible efecto en rendimiento y calidad del grano.

5.- Es una variedad de buena caña y sin problemas de tendedura; se recomienda no aplicar regulador de crecimiento.

\section{CONCLUSION}

Pionero INIA es producto de una cruza compuesta, donde sus progenitores portan genes de especies ancestrales como ser Triticum dicoccum (AABB), lo que permite la existencia de 
una mayor diversidad genética en su patrimonio genético. Esto es particularmente importante ya que la creciente uniformidad genética de las variedades cultivadas actuales, combinada con los efectos del cambio climático, hace que los cultivos resulten más vulnerables al estrés biótico y especialmente abiótico, como la sequía, el calor y la salinidad, siendo estos de gran relevancia para la producción de trigo. Por tanto, esta variedad, dada sus características originarias (progenitores silvestres), tiene el potencial para transferir la variabilidad deseada relacionada a la adaptabilidad a los estrés ambientales, una mayor resistencia genética a las enfermedades y mayores potenciales productivos, lo cual podría contribuir a mejorar sustancialmente la genética del actual trigo chileno, y ampliar su zona de adaptación y siembra. De acuerdo a los resultados presentados, es un trigo de doble propósito, con gran potencial productivo, sanidad y adecuadas características nutricionales, por lo que se considera como una nueva y potente alternativa para el sur de Chile, siendo esta la primera en su género.

\section{LITERATURA CITADA}

Bushuk, W., and R. Zillman. 1978. Wheat cultivar identification by gliadin electrophoregrams. I. Apparatus, method and nomenclature. Can. J. Plant Sci. 58:505-515.

Hevia, F., R. Villegas, y H. Tollenaar. 1986. Influencia de algunas características físicas del grano de almidón sobre la calidad panadera del trigo. Agro-Ciencia 2(1):27-38

INE. 2014. Informe Anual Agropecuario. ISBN: 978-956-323-168-. 153p. Instituto Nacional de Estadísticas (INE), Santiago, Chile.

Instituto Nacional de Normalización. 2000. Norma Chilena. Nch 1237-2000. Trigo Harinero - Requisitos. 16 p. Instituto Nacional de Normalización, Santiago, Chile.

Jobet, C., R. Madariaga, I. Matus, R. Campillo, y J. Zúñiga. 2017. Maxwell: El cultivar de trigo que ha superado las barreras productivas en el sur de Chile. 2017. Chilean J. Agric Anim. Sci., ex Agro-Ciencia 33(1):39-44.

Mellado, M. 2007. El trigo en Chile. Cultura, Ciencia y Tecnología. Colección Libros INIA, $\mathrm{N}^{\circ}$ 21. ISBN956-7016-28-3. $684 \mathrm{p}$. Instituto de Investigaciones Agropecuarias (INIA), Quilamapu, Chillán, Chile.

ODEPA. 2017. Estadísticas Productivas. Disponible en http://www.odepa.gob.cl/ (Consulta 11 de mayo de 2017).
Payne P., and G. Lawrence. 1983. Catalogue of alleles for the complex gene loci, Glu-A1, Glu-B1, and Glu-D1 which code for the high molecular- weight subunits of glutenin in hexaploid wheat. Cereal Res. Comm. 11: 2935.

Peterson, R., J. Campbell, and A. Hannah. 1984. A diagrammatic scale for estimating rust intensity of leaves and stem of cereals. Can. J. Res. Sect. C 26:496-500.

Saari, E., and J. Prescott. 1975. A scale for appraising the foliar intensity of wheat diseases. Plant Dis. Rep. 59:377-380.

Singh, N., K. Shepherd, and G. Cornish. 1991. A simplified SDS-PAGE procedure for separating LMW subunits of glutenin. J. Cereal Sci. 14:203-208.

Zúñiga, J., C. Jobet, H. Campos, M. Mathías, y D. Schäfer. 2001. Identificación molecular de alelos de puroindolinas en trigo (Triticum aestivum L.). p. 150. IV Encuentro Latinoamericano de biotecnología vegetal, Goiania, Brasil. 04 al 08 de junio del 2001. REDBIO. Instituto Interamericano de Cooperación para la Agricultura (IICA), Goiania, Brasil.

Zúñiga, J., C. Jobet, H. Campos, P. Rathgeb, y A. Arcos. 2000. Identificación molecular de genotipos translocados en trigo (Triticum aestivum L.). p. 56. XXXIII Reunión Anual Sociedad de Genética de Chile, Concepción, Chile. 11 al 13 de octubre del 2000. Universidad de Chile, Santiago, Chile. 